J. Lake Sci. (湖泊科学) , 2007, 19(3) :289-298

http:// www. jlakes. org. E-mail: jlakes@ niglas. ac.cn

(c) 2007 by Journal of Lake Sciences

\title{
东洞庭湖保护区大型底栖动物空间分布格局及水质评价
}

\author{
谢志才 $^{1}$, 张君倩 ${ }^{1,2}$, 陈 静 ${ }^{1,2}$,马 凯 ${ }^{1}$, 刘瑞秋 ${ }^{1}$, 汪亚平 ${ }^{1}$, 蔡庆华 ${ }^{1}$ \\ ( 1 : 淡水生态与生物技术国家重点实验室,中国科学院水生生物研究所, 武汉 430072) \\ (2: 中国科学院研究生院, 北京 100039)
}

摘 要:2001 年 9 月对东洞庭湖保护区大型底栖动物进行调查, 共记录动物 4 门 8 纲 20 科 51 种. 其中, 骞毛类 2 科 18 种 (35.3\%), 软体动物 8 科 15 种 $(29.4 \%)$, 水生昆虫 7 科 14 种 $(27.5 \%)$, 其他动物 4 种 $(9.1 \%)$. 软体动物是东洞庭湖底 栖动物的最主要类群 (占总丰度的 $71.1 \%$ ). 其中, 腹足类的长角涵螺 (Alocinma longicornis)、铜锈环棱螺 (Bellamya aerugi$n o s a$ )、中华沼螺 (Parafossarulus sinensis)、纹沼螺 (P. striatulus) 和塞毛类的苏氏尾鳃蚂 (Branchiura sowerbyi) 为该保护区的 优势种. 样点间平均物种丰富度 6.3, 三种多样性指数 (Margalef, Simpson 和 Shannon) 平均值分布为 1.38、4.05 和 1.47. 刮食者和直接收集者为底栖动物最重要的摄食类群(占总密度的 $66.7 \%$ 和 $19.9 \%$ ). 结合 GPS, 将野外调查数据构建 GIS 数据库. 基于 GIS 软件平台, 运用 Kringing 插值法,构建动物丰度、生物多样性及污染生物指数 (BI) 等的空间分布格局, 较为直观地反映了保护区底栖动物的分布情况及污染状况. 提取出主要生物类群 (软体动物、塞毛类和摇蚊幼虫) 和 5 种 优势种分布的高浓度块,显示塞毛类与其它两类的分布基本不重叠,主要分布在保护区的东南和西北的深水区域. 进一 步分析检验各主要分布区域的 16 种水体理化指标, 发现水深是影响保护区底栖动物分布的关键因子, 而硫酸盐则主要制 约塞毛类的分布格局, 硬度是影响软体动物分布的主要因子. 全湖平均 Hilsenhoff 生物指数 $(B I)$ 值 8.18 , 指示保护区承受 较高的有机污染, 模拟生成的 $B I$ 分布图显示, 西南部的深水区域、北部及东南部的局部区域水质相对较好, 而东北部的沿 岸带大部分水体及南部近六门闸的部分区域水体水质相对较差.

关键词: 东洞庭湖保护区; 大型底栖动物;群落结构;功能摄食类群;空间分布格局;GIS

\section{Spacial distributional pattern of macrozoobenthos and pollution evaluation in East Lake Dongting Reserve}

\author{
XIE Zhicai ${ }^{1}$, ZHANG Junqian ${ }^{1,2}$, CHEN Jin ${ }^{1,2}$, MA Kai ${ }^{1}$, LIU Ruiqiu ${ }^{1}$, WANG Yaping $^{1}$ \& CAI Qinghua ${ }^{1}$ \\ (1: State Key Laboratory of Freshwater Ecology and Biotechnology, Institute of Hydrobiology, Chinese Academy of \\ Sciences, Wuhan 430072 , P. R. China) \\ (2: Graduate School of the Chinese Academy of Sciences, Beijing 100039,P. R China)
}

\begin{abstract}
Investigation of macrozoobenthos was carried out in East Lake Dongting Reserves in September, 2001. All total, 51 taxa were identified from thirty-two sampling sites, of which, 18 oligchaetes, 15 mollusks, 14 insects and 4 other forms. Mollusks constituted the most dominant group and accounted for more than $70 \%$ of the total abundance. Among which, four gastropods, Alocinma longicornis, Bellamya aeruginosa, Parafossarulus sinensis, P. striatulus and one oligochaete Branchiura sowerbyi were the dominant species. The richness and three biodiversity index values ( (Margalef, Simpson and Shannon) per site were 6.3, 1.38, 4.05 and 1.47, respectively. Assemblages were composed mainly of scrapers $(66.7 \%)$ and collector-gatherers $(19.9 \%)$, and to a lesser extent to other functional feeding groups.

The investigation data were compiled and a GIS database of macrozoobenthos was established. A vector boundary map of the lake was digitized and georeferenced, then used to introduce sampling sites. Under the support of

* 国家自然科学基金项目 (30370250;30070153), 国家重点基础发展规划 (973) 项目 (2002CB412310), 中国科学院 知识创新工程重大项目 (KZCX12SW212) 和 863 项目 (2004AA213123) 资助联合. $2006-04-17$ 收稿; $2006-11$ 13 收修改稿. 谢志才,男,1967 年生,副研究员,博士.
\end{abstract}


GIS software Arcview 3.2 and the relative extension modules (Spatial Analyst Extension 2. 0 and Kringing Interpolator Extension 3.22 SA), the GIS database was employed to simulate spacial distribution patterns of parameters of macrozoobenthos and Hilsenhoff biotic index (BI). The simulation process was based on the principle of Kringing interpolation technique. The simulated results were stored as Arcview Grid format with a grid precision of $20 \mathrm{~m}$. The higher distributional pattern of main groups and five dominated species were overlapped based on the $2-4$ times as mean abundance, and the 16 physical-chemical parameters were collected in these higher distributional regions.

The simulated patterns of macrozoobenthos showed that these patterns were relatively high spatial heterogeneity, and distributions of most parameters of macrozoobenthos had usually $2-4$ high value distributed zones in the whole lake. The oligochaete pattern showed basically no overlapping with two other groups, and mainly distributed in deeper region. ANOVA detected water depth was the key factor influencing the distribution patterns of macrozoobenthos, and $\mathrm{SO}_{4}^{2-}$ mainly controlling the pattern of oligochaete, and hardness the main factor affecting mollusks. The average of BI (8.18) revealed the Reserves endured higher organic pollution.

Keywords : East Lake Dongting Reserves; macrozoobenthos; community structure; functional feeding groups; spacial distribution pattern; GIS

洞庭湖为我国第二大淡水湖,位于长江中游荆江南岸, 属过水性吞吐型湖泊; 南纳湘、资、沅、澧四水而 北通长江. 现有天然湖泊面积 $2691 \mathrm{~km}^{2}$, 洪道面积 $1307 \mathrm{~km}^{2}$, 最大容积 $187.4 \times 10^{8} \mathrm{~m}^{3}$ (城陵矶水位 34.03 $\mathrm{m})$. 流速 $0.00-1.05 \mathrm{~m} / \mathrm{s}$, 常年泥沙含量为 3.3-610.3 mg/L, 湖泊多年平均径流量 $3126 \times 10^{8} \mathrm{~m}^{3}$. 属亚热 带气候区, 年降雨量 $1100-1400 \mathrm{~mm}$, 年均湿度 $80 \%$, 无霜期 $273-276 \mathrm{~d}$, 年日照 $1700-1800 \mathrm{~h}$, 年均气温 $16.4-17.0^{\circ} \mathrm{C}{ }^{[1]}$. 洞庭湖是我国重要的湿地之一, 具有调蓄洪水、调节气候、涵养水源、净化水质、维护生物 多样性等多种生态和环境功能. 近年来, 随着湖滨人口增长, 对资源的过度开发和围湖垦殖加剧、湖底泥沙 淤积严重导致的湿地骤增、土地无节制的利用等多种原因, 导致湖泊的生态平衡多已遭到不同程度的破坏， 严重抑制这些水体综合生态功能的发挥. 而洪涝灾害次数增多, 频率加快, 是近年来洞庭湖生态系统病症 的突出表征 ${ }^{[2]}$.

东洞庭湖保护区 $\left(28^{\circ} 59^{\prime}-29^{\circ} 38^{\prime} \mathrm{N}, 112^{\circ} 43^{\prime}-113^{\circ} 15^{\prime} \mathrm{E}\right)$ 位于长江中游荆江江段南侧、洞庭湖北部,地 处湖南省东北部. 北起长江湘鄂两省主航道分界线, 南至否石山, 总覆盖面积 $190 \mathrm{~km}^{2}$. 该保护区是侯鸟 的理想越冬场所. 经记录的水禽约 200 余种,每年约有 200 万只鸟类在此越冬. 其中有国家一级保护鸟类 如白鹳 (Ciconia aconia)、白头鹤 (Grus monacha)、白鹤 (Otis tarda) 等 6 种, II 级保护鸟类 26 种. 已记录的鱼 类有 12 目 23 科 114 种, 其中濒危的鱼类有白鲟(Psephurus gladius) 和中华鲟 (Acipenser sinensis) 等 ${ }^{[3]}$.

洞庭湖底栖动物的研究鲜有报道 ${ }^{[4]}$. 本研究在探明东洞庭湖保护区底栖动物群落结构的基础上, 尝试 应用 GIS 技术构建底栖动物的空间分布格局, 并结合底栖动物数据, 评价保护区水质污染状况, 模拟该区域 的水质的有机污染状况的直观的分布图,期望为治理和保护该保护区提供科学数据.

\section{1 材料与方法}

\section{1 大型底栖动物的采集}

2001 年 9 月 17-29 日对东洞庭湖保护区进行大型底栖动物野外调查. 共设 32 个样点(图 1). 定量采 集用 $1 / 16 \mathrm{~m}^{2}$ 改良的彼德生氏采泥器进行, 每站样点采 2 次重复样, 泥样经 $450 \mu \mathrm{m}$ 的铜篮洗净后, 用肉眼在 解剖盘中将动物标本拣出, 标本用 $4 \%$ 甲醛溶液固定. 在实验室中将标本鉴定至尽可能低的分类单元, 然后 计数和称重,换算成单位面积的含量.

功能摄食类群的确定参阅相关的文献 ${ }^{[5-7]}$.

各样点生物多样性的计算采用 Shannon-Wiener 指数、Simpson 指数和 Margalef's 指数.

Margalef's index $(d)=(S-1) / \ln N$

Simpson index $(D)=1 /\left\{\sum\left(n_{i} / N\right)^{2}\right\}$ 


$$
\text { Shannon-Wiener index }\left(H^{\prime}\right)=-\sum\left\{\left(n_{i} / N\right) \ln \left(n_{i} / N\right)\right\}
$$

式中, $S$ 为总物种数, $N$ 为总密度, $n_{i}$ 为物种 $\mathrm{i}$ 的密度.

用修订的 Hilsenhoff 生物指数 $(B I)$ 平均各站点的有机污染状况:

$$
B I=\sum x_{i} t_{i} / n
$$

式中, $x_{i}$ 为物种 $i$ 的个体数, $t_{i}$ 为物种 $i$ 的耐受值, $n$ 为总个体数.

水质评价标准为: $B I=0.00-3.75$ 极清洁; $3.76-4.25$ 很清洁; $4.26-5.00$ 清洁;5.01-5.75一般;

$5.76-6.50$ 轻度污染; $6.51-7.25$ 污染; $7.26-10.00$ 严重污染 $^{[8,9]}$.

\subsection{GIS 数据库构建}

利用 Calcomp DrawingBoard III 型数字化仪数字化得到东洞庭湖保护区轮廓图 (1:90000). 参照 1: 1000000中国基础地理信息图水系图层完成地面校正 (Georeference),赋予轮廓图地理信息.

将野外调查及实验分析数据按样点编号输人计算机, 同时一一对应从 GPS 导人每样点的地理坐标. 在 ArcView 3.2 平台支持下, 以数字化的轮廓图为底图, 通过地理坐标定位生成湖泊中真实的样点分布图. 分 布图层链接两个数据库文件, 文件为数据库通用软件 Foxpro 支持的 DBF 格式. 其中, 第一个数据库为洞庭 湖底栖动物数据库,包含每样点的物种组成和每种现存量等信息; 第二个数据库为洞庭湖理化因子数据库, 包括各样点的水环境各理化因子的实验分析结果. 数据库通过样点编号和样点分布图层相连. 结合保护 区底栖动物的分布特点, 以每样点底栖动物的各指标 (总丰度及各主要类群丰度、优势种丰度、三种生物多 样性指数及 $B I$ 指数) 为变量, 利用 Kringing 插值法进行内插运算, 模拟生成保护区底栖动物格局及 $B I$ 分布 图. 分布图格式为 ArcView 栅格图 (Grid) 格式,栅格精度为 $20 \mathrm{~m}$.

底栖动物主要类群和优势种集中分布区域分布格局的提取按其平均密度的 $2-3$ 倍进行,然后进行叠 加. 从两个 GIS 数据库中提取大型底栖动物信息和理化因子信息, 利用 GIS 空间分析中的插值技术功能提 取出底栖动物主要类群及优势种集中分布区域的 16 种理化因子, 应用方差分析比较它们的差异, 以期探明 影响保护区底栖动物分布格局的关键因子.

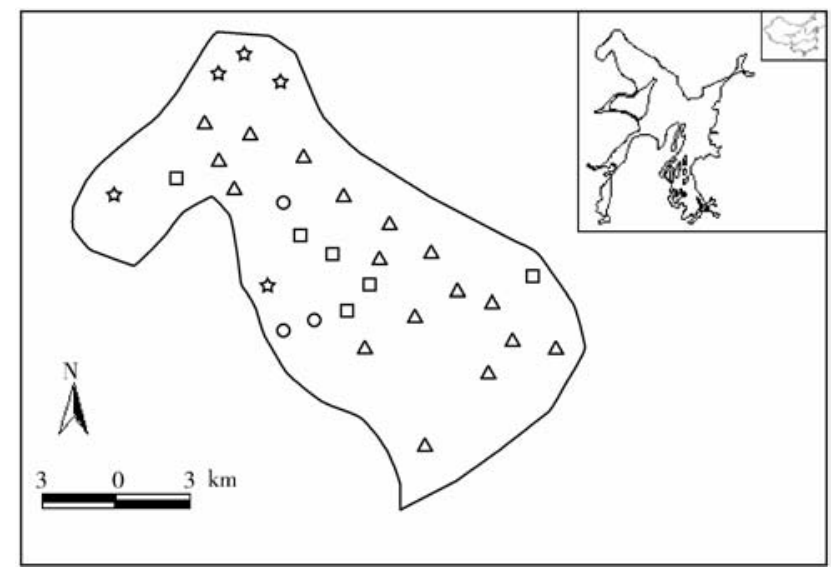

图 1 东洞庭湖保护区底栖动物样点分布图

Fig. 1 Macrozoobenthic sampling sites of the East Lake Dongting Reserve

\section{2 结果}

\section{1 群落结构}

共发现底栖动物 51 种, 隶 4 门 8 纲 20 科. 其中,塞毛类 2 科 13 属 18 种 (35.3\%), 软体动物 8 科 13 属 15 种 $(29.4 \%)$ ), 摇蚊科 8 属 8 种 ( $15.7 \%$ ), 其它昆虫 6 科 6 属 6 种 $(11.8 \%$ ), 其他动物 8 种 $(9.1 \%)$ (图 $2)$. 样点间平均物种丰富度 6.3. 软体动物为保护区底栖动物的绝对优势类群, 其密度 $\left(193.0 \mathrm{ind} . / \mathrm{m}^{2}\right)$ 占 总密度的 $71.1 \%$, 其次为葟毛类 $\left(49.0\right.$ ind. $\left./ \mathrm{m}^{2}, 18.1 \%\right)$ 和水生昆虫 $\left(20.5 \mathrm{ind} . / \mathrm{m}^{2}, 8.0 \%\right)$. 生物量方面, 
软体动物占了绝大部分 $\left(173.488 \mathrm{mg} / \mathrm{m}^{2}, 99.9 \%\right.$ ) (表 2). 优势度分析表明, 腹足类的长角涵螺 (A. longicornis ) 为第一优势种 $\left(85.5 \mathrm{ind} / \mathrm{m}^{2}, 31.4 \%\right)$, 其次为铜锈环棱螺 (B. aeruginosa) $\left(35.0 \mathrm{ind} . / \mathrm{m}^{2}, 12.7 \%\right)$ 、中 华沼螺 $(P$. sinensis $)\left(29.0\right.$ ind. $/ \mathrm{m}^{2}, 10.7 \%$ )、纹沼螺 $(P$. striatulus $)\left(15.0\right.$ ind. $\left./ \mathrm{m}^{2}, 5.5 \%\right)$ 和寡毛类的苏 氏尾鳃蚓 (B. sowerbyi) (14.0 ind. $\left./ \mathrm{m}^{2}, 5.2 \%\right)$. 功能摄氏类群研究显示, 洞庭湖底栖动物的物种组成主要 是直接收集者 (21 种, 占总物种数的 $41.2 \%$ ), 其次是捕食者 (11 种, $21.6 \%$ ), 刮食者 $(10$ 种, 19.6\%), 过 滤收集者 (6 种, $11.8 \%$ ) 和撕食者 (3 种, 5.9\%). 而刮食者为底栖动物的最重要的摄食类群 $(66.7 \%)$, 其 次是直接收集者 $(19.9 \%) 、$ 捕食者 $(7.2 \%)$ 、过滤收集者 $(4.8 \%)$ 和撕食者 $(1.5 \%)$.

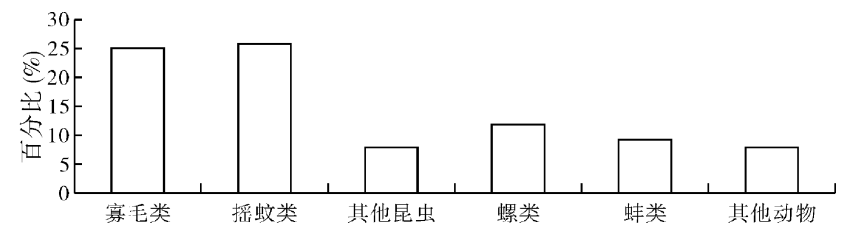

图 2 底栖动物各类群物种数的比较

Fig. 2 Comparison of different group taxa number in Reserve

表 2 东洞庭湖保护区底栖动物各类群的平均丰度 (密度: ind $/ \mathrm{m}^{2}$; 生物量: $\mathrm{g} / \mathrm{m}^{2}$ )

Tab. 2 Mean standing crops of different groups in the Reserve

\begin{tabular}{ccccccc}
\hline & 密度 & 范围 & 标准误 & 生物量 & 范围 & 标准误 \\
\hline 寡毛类 & 49.0 & $0.0-208.0$ & 59.4 & 0.064 & $0.000-0.575$ & 0.123 \\
水生昆虫 & 20.5 & $0.0-96.0$ & 30.8 & 0.037 & $0.000-0.504$ & 0.977 \\
软体动物 & 193.0 & $16.0-624.0$ & 159.1 & 173.488 & $0.253-1093.840239 .191$ \\
其他动物 & 9.0 & $0.0-64.0$ & 15.2 & 0.125 & $0.000-3.328$ & 0.587 \\
合计 & 271.5 & $0.5-85.5$ & 13.2 & 173.714 & $0.000-257.774$ & 100.042 \\
\hline
\end{tabular}

表 3 东洞庭湖底栖动物各功能摄氏类群的物种数及丰度 (密度: $i n d / \mathrm{m}^{2}$; 生物量: $\mathrm{g} / \mathrm{m}^{2}$ )

Tab. 3 Taxa richness, mean density and biomass of different feeding groups in East Lake Dongting Reserve

\begin{tabular}{ccccccc}
\hline & 物种数 & $\%$ & 密度 & $\%$ & 生物量 & $\%$ \\
\hline 过滤收集者 & 6 & 11.8 & 13.0 & 4.8 & 71.470 & 41.1 \\
直接收集者 & 21 & 41.2 & 54.0 & 19.9 & 0.180 & 0.1 \\
捕食者 & 11 & 21.6 & 19.5 & 7.2 & 0.023 & 0.01 \\
刮食者 & 10 & 19.6 & 181.0 & 66.7 & 102.018 & 58.7 \\
撕食者 & 3 & 5.9 & 4.0 & 1.5 & 0.022 & 0.01 \\
合计 & 51 & 100.0 & 271.5 & 100.0 & 173.714 & 100.0 \\
\hline
\end{tabular}

\section{2 大型底栖动物的分布格局}

\section{2 .1 总现存量及各主要类群现存量的分布格局}

图 3 是模拟出的东洞庭湖保护区底栖动物总密度和生物量的分布格局. 可以看出, 底栖动物总现存量 (密度和生物量) 的分布具有明显的空间异质性, 出现数个高浓度斑块. 其中总密度出现 5 个峰点, 最高值 位于东北部沿岸带 $\left(800 \mathrm{ind} / \mathrm{m}^{2}\right)$, 总生物量出现 3 个峰点, 最高值位于东北部亚沿岸带 $\left(257.774 \mathrm{~g} / \mathrm{m}^{2}\right)$. 进 一步分析各主要类群 (寡毛类、摇蚊科幼虫和软体动物) 现存量的分布格局 (图 4), 显示, 底栖动物的分布 格局与软体动物的较相似, 表明洞庭湖底栖动物的分布格局主要由软体动物所主导 (即软体动物是洞庭湖 保护区最主要的底栖动物生物类群). 
进一步提取出主要生物类群 (软体动物、寡毛类和摇蚊幼虫) 密度分布的高浓度块, 然后进行叠加, 得 图 5A. 可以看出, 寡毛类的分布与其它两类基本不重叠, 其主要分布区域在东南和西北的深水区域, 而软 体动物和摇蚊幼虫的分布在南部有部分的重叠区域. 三种类群主要分布区域的 16 种理化指标方差分析表 明, 仅水深和硬度在各类群的集中分布区域内达到显著差异水平 $(P<0.05)$ (表 4$)$. 表明水深是影响洞庭 湖保护区底栖动物分布格局的最重要的环境参数,而硬度则是限制软体动物分布的主要因素.

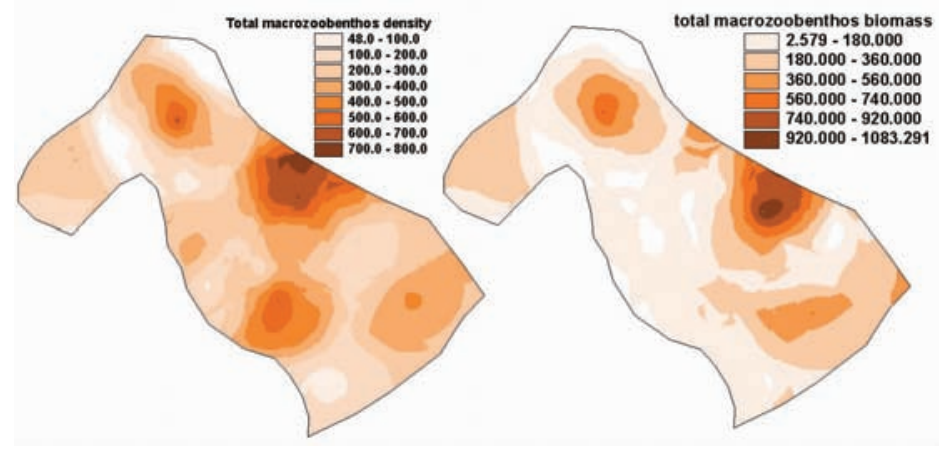

图 3 洞庭湖保护区底栖动物密度和生物量分布格局 ( A: 密度; B:生物量)

Fig. 3 Distribution patterns of density and biomass of macrozoobenthos in East Dongting Reserve( A: density; B: biomass)

2.2 .2 优势种类的分布格局 模拟出的 5 种优势种的分布格局显示 (图 6 ), 长角涵螺、大沼螺和苏氏尾鳃蚓 在全湖的分布格局相对均匀, 长角含量仅在东北部沿岸局部区域有一高浓度块, 其最高密度为 $505.2 \mathrm{ind} /$ $\mathrm{m}^{2}$ 和最高生物量 $60.960 \mathrm{~g} / \mathrm{m}^{2}$. 大沼螺则有 2 个高浓度区域, 其最高值分别为 $375.7 \mathrm{ind} / \mathrm{m}^{2}$ 和 $142.508 \mathrm{~g} /$ $\mathrm{m}^{2}$. 相比较而言, 铜锈环棱螺和中华沼螺的分布较不规则, 铜锈环棱螺出现 4 个密度高峰和 3 个生物量高 峰, 其最高值分布在东北角的亚沿岸带, 为 $127.6 \mathrm{ind} / \mathrm{m}^{2}$ 和 $217.20 \mathrm{~g} / \mathrm{m}^{2}$; 而中华沼螺在东北部沿岸区即西 南部局部区域相对较高,最高值为 $96.2 \mathrm{ind} / \mathrm{m}^{2}$ 和 $14.52 \mathrm{~g} / \mathrm{m}^{2}$.

进一步提取出以上 5 种优势种密度分布的高浓度块, 然后进行叠加, 得图 5B. 显示苏氏尾鳃蚓与其余 4 种完全不重叠, 主要分布在东南部和西部的局部深水区域. 而其余四种腹足类分布均有不同程度的重叠. 其中, 长角涵螺与中华沼螺的分布区域重叠, 而环棱螺和大沼螺有部分区域重叠. 16 种理化指标的方差分 析表明,仅水深和硫酸盐在各类群的集中分布区域内达到显著差异水平 $(P<0.05)($ 表 4$)$. 表明水深和硫 酸盐是影响东洞庭湖保护区底栖动物 5 优势种分布的主要因子. 将苏氏尾鳃蚓与 4 种软体动物的水体理 化参数进行 $t$ 检验, 结果显示水深存在显著差异 $(P<0.05)$. 表明, 与其余 4 种软体动物比较而言, 苏氏尾 鳃蚓更喜在水体相对较深、硫酸盐含量较高的的环境生存. 4 种软体动物集中分布区域主要理化参数的 $t$ 检验结果显示, 水深亦是软体动物分布的最主要的环境因子, 长角涵螺和中华沼螺主要分布在沿岸带的浅 水区域,而环棱螺和大沼螺则主要分布在亚沿岸带的深水区域.

2.2 .3 生物多样性的分布格局和水质评价初探 表 5 给出保护区底栖动物三种生物多样性指数和 Hilsenhoff' s 生物指数 $(B I)$ 值. 可以看出, 保护区底栖动物多样性指数相对较低. 构建的底栖动物 3 种生物多样性 指数的分布格局显示 (图 7), 底栖动物生物多样性的高浓度区域主要集中在西南部的深水区域, 在北部和 南部的局部区域亦有 2 个峰值.

计算出全湖的 Hilsenhoff' s 生物指数 $(B I)$ 均值为 8.18 (表 5), 反映保护区有较高的有机污染. 模拟的 Hilsenhoff's 生物指数 $(B I)$ 分布格局则与 3 种生物多样性分布基本呈相反的分布(图 7 ). 除保护区西南部 的深水区域、北部及东南部的局部区域水质为轻度污染 $(B I \leqslant 6.50)$ 或污染水质 $(B I$ 介于 $6.50-7.25$ 之间) 外, 全湖大部分区域水体均为严重污染 $(B I>7.26)$, 其中东北部的沿岸带大部分水体及南部近六门闸的部 分区域水体水质最差 $(B I>9.00)$. 

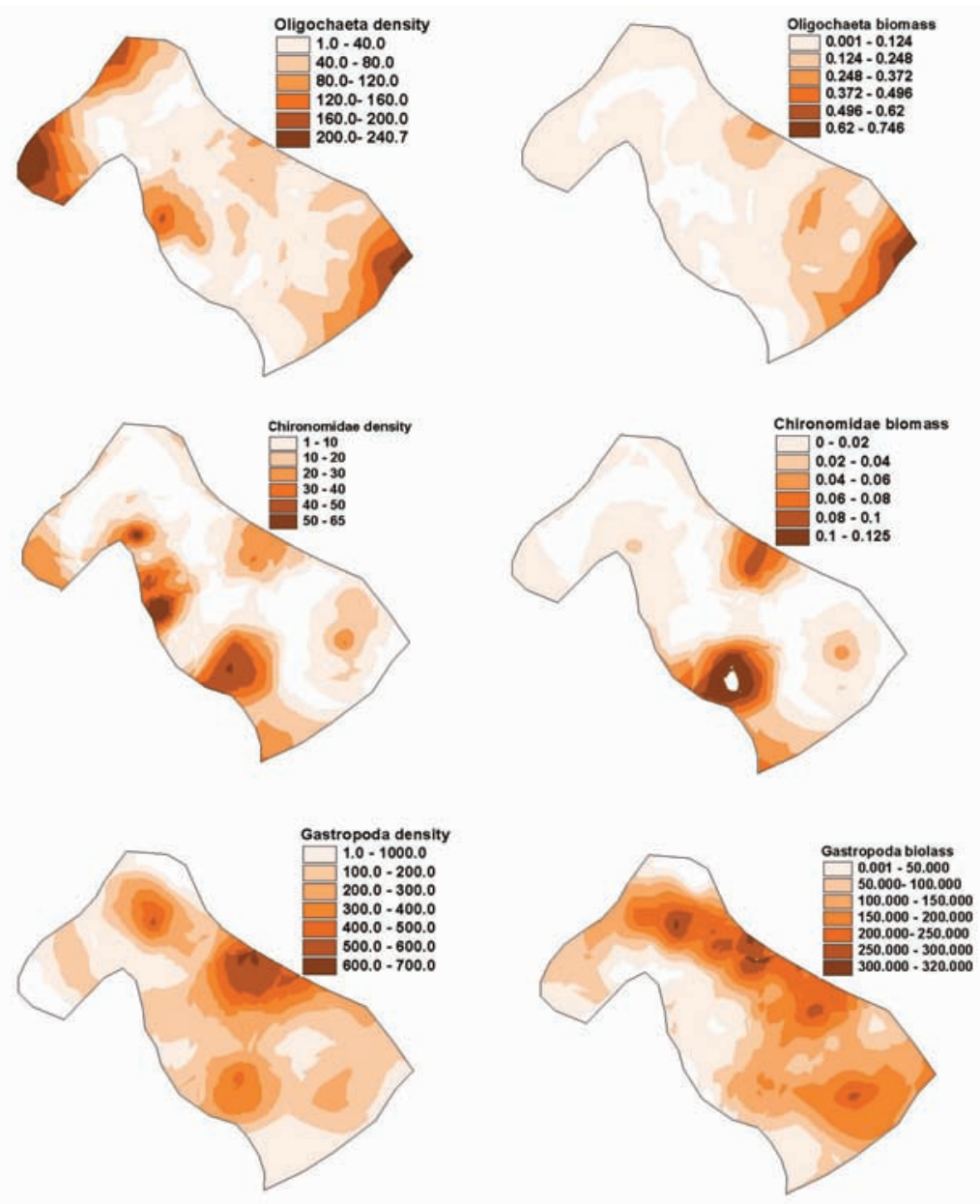

图 4 保护区底栖动物各主要类群密度和生物量分布格局 ( A, B : 臭毛类; C, D: 摇蚊科幼虫; $\mathrm{E}, \mathrm{F}$ : 软体动物)

Fig. 4 Distribution patterns of densities and biomasses of main macrobenthic groups in the Reserve ( A, B: oligochaetes; C, D : chironomids: E, F : mollusks).

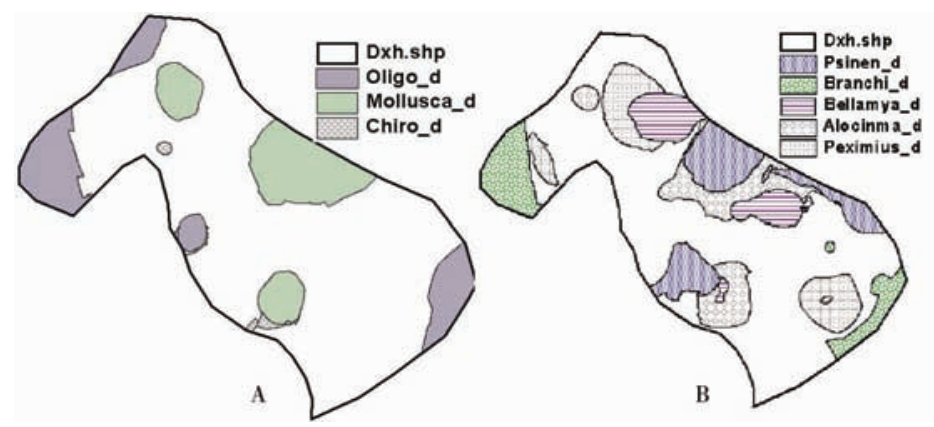

图 5 保护区底栖动物主要类群及 5 种优势种集中分布区域的密度分布格局 ( A: 主要类群; B: 优势种)

Fig. 5 Main density distribution patterns of main groups and dominated species in the Reserve ( A: main groups; B: dominated species) 
表 4 底栖动物主要类群及 5 种优势种主要分布区域 16 种理化指标的方差分析

Tab. 4 ANOVA analysis of physical-chemical parameters in main distributional regions

of main groups and dominated species

\begin{tabular}{|c|c|c|c|c|}
\hline & \multicolumn{2}{|c|}{ 主要类群 } & \multicolumn{2}{|c|}{5 种优势种 } \\
\hline & $F$ 值 & $P$ 水平 & $F$ 值 & $P$ 水平 \\
\hline 水深 $(\mathrm{m})$ & 4.454 & $0.030^{*}$ & 5.78 & $0.002 *$ \\
\hline 透明度 ( m) & 0.725 & 0.501 & 0.895 & 0.482 \\
\hline 电导率 $(\mu \mathrm{s} / \mathrm{L})$ & 0.410 & 0.671 & 0.379 & 0.821 \\
\hline $\mathrm{pH}$ & 0.509 & 0.6111 & 0.32 & 0.290 \\
\hline $\mathrm{SO}_{4}^{2-}(\mathrm{mg} / \mathrm{L})$ & 1.432 & 0.270 & 5.91 & $0.002 *$ \\
\hline $\mathrm{NO}_{2}^{-}(\mathrm{mg} / \mathrm{L})$ & 1.506 & 0.253 & 0.622 & 0.651 \\
\hline $\mathrm{SiO}_{2}(\mathrm{mg} / \mathrm{L})$ & 2.716 & 0.098 & 0.528 & 0.716 \\
\hline $\mathrm{PO}_{4}^{3-}(\mathrm{mg} / \mathrm{L})$ & 0.265 & 0.771 & 0.686 & 0.608 \\
\hline $\mathrm{NH}_{4}^{+}(\mathrm{mg} / \mathrm{L})$ & 0.710 & 0.507 & 0.117 & 0.975 \\
\hline $\mathrm{TN}(\mathrm{mg} / \mathrm{L})$ & 1.940 & 0.1781 & 0.45 & 0.249 \\
\hline $\mathrm{TP}(\mathrm{mg} / \mathrm{L})$ & 0.335 & 0.720 & 0.571 & 0.686 \\
\hline 碱度 $(\mathrm{mg} / \mathrm{L})$ & 1.212 & 0.325 & 0.744 & 0.571 \\
\hline 硬度 & 4.673 & $0.026^{*}$ & 2.09 & 0.114 \\
\hline $\mathrm{Ca}^{2+}(\mathrm{mg} / \mathrm{L})$ & 2.326 & 0.132 & 1.14 & 0.362 \\
\hline $\mathrm{Cl}^{-}(\mathrm{mg} / \mathrm{L})$ & 1.667 & 0.222 & 0.168 & 0.953 \\
\hline $\operatorname{COD}(\mathrm{mg} / \mathrm{L})$ & 1.787 & 0.201 & 0.985 & 0.434 \\
\hline
\end{tabular}

*表示显著.

表 5 保护区底栖动物 3 种多样性指数及 Hilsenhoff 生物指数

Tab. 5 The value of three biodiversity indices and Hilsenhoff biotic index in the Reserve

\begin{tabular}{ccccc}
\hline & 均值 & 最大值 & 最小值 & 标准误 \\
\hline Margalef's index & 1.38 & 2.65 & 0.28 & 0.54 \\
Simpson index & 4.05 & 9.28 & 1.18 & 1.89 \\
Shannon-Wiener index & 1.47 & 2.39 & 0.29 & 0.47 \\
Hilsenhoff biotic index $(B I)$ & 8.18 & 9.27 & 6.00 & 0.65 \\
\hline
\end{tabular}

\section{3 讨论}

与静水湖泊相比,在保护区出现一些适应流水或半流水环境的物种,典型的如寡毛类的湖沼管水蚓 (A. limnobius)、厚唇嫩丝蚓 ( T. mastix)、半翅目的划春科 (Corixidae)、微黾科 (Hebridae)、鞘翅目的沼梭科 (Haliplidae) 、摇蚊科的短小流水长蚹摇蚊 (R. exiguus) 及腹足纲的钉螺 (O. hupensis) 和双壳纲的江蚬 ( $C$. fluminalis $)$ 等, 反映东洞庭湖保护区底栖动物的物种组成表现出适应流水生境的特征.

基于 GIS 构建的底栖动物的分布格局,较为直观地反映东洞庭湖保护区底栖动物的空间分布格局. 底 栖动物的不同指标在全湖的分布除少数指标出现一个高浓度峰值, 其余地区较为变化平缓外, 多数指标均 出现数个浓度峰点, 指示底栖动物表现出较强的空间异质性. 模拟的底栖动物主要生态类群的分布显示, 保护区底栖动物的分布主要由腹足类所左右, 表明腹足类是保护区的主要类群.

一般认为,在浅水草型湖泊中,水草是影响底栖动物分布的最为重要的环境因子 ${ }^{[10-12]}$. 高度繁茂的高 等植物 (主要是沉水植物) 为底栖动物提供了更加多样的生境, 利于底栖动物生活、摄食和繁殖 ${ }^{[13,14]}$, 水草 上附殖的丰富的着生藻类、小型动物和有机碎屑,给底栖动物如腹足类、昆虫等提供丰富的食物来源, 而繁 盛的水草亦能使底栖动物有效地逃避鱼类和其它大型动物的捕食以及水流、风力等造成的不利影 

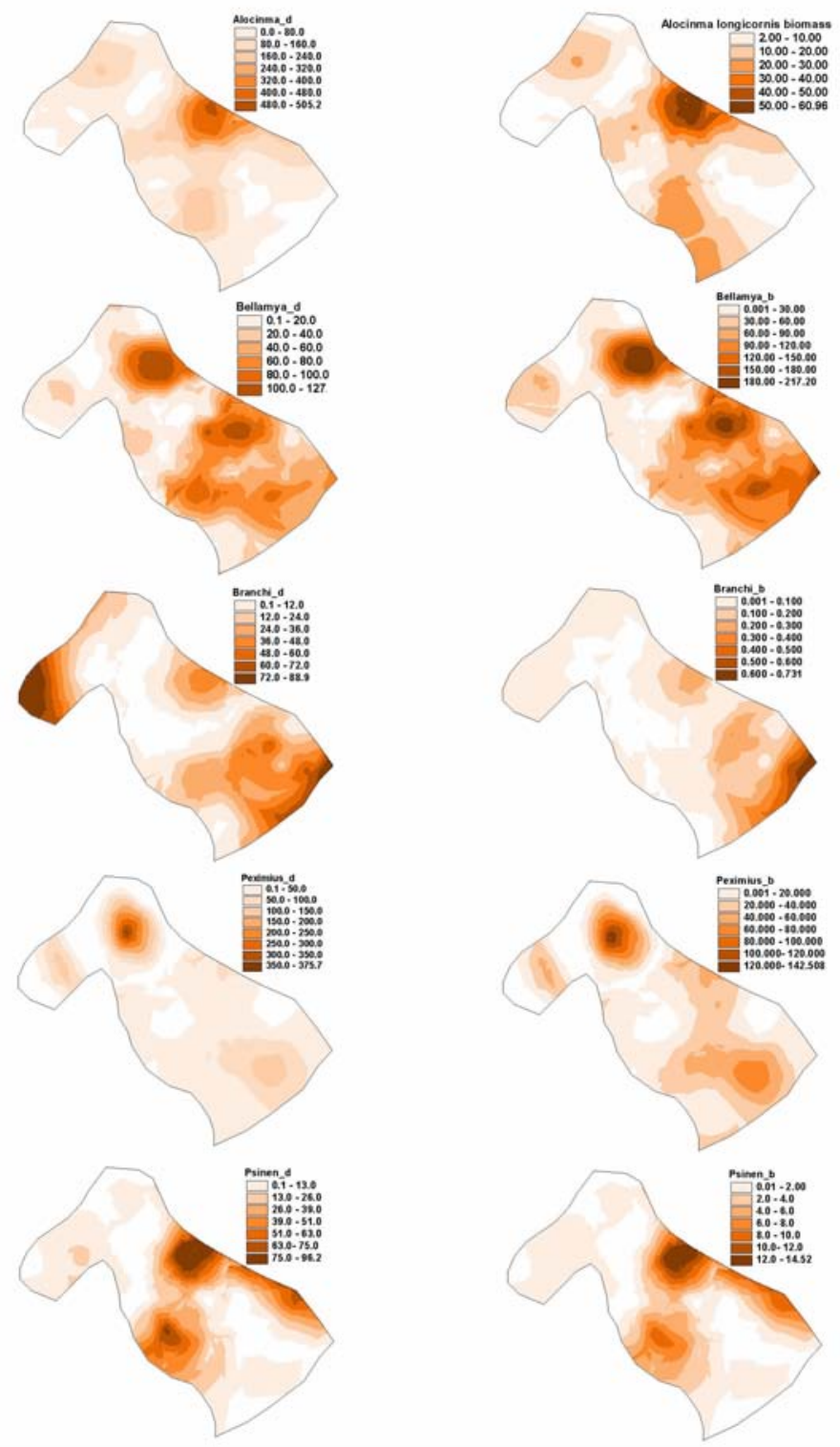

图 6 保护区底栖动物优势种密度和生物量分布格局图. A, B : 长角涵螺; C, D : 铜锈环棱螺; E, F : 苏氏尾鳃蚓; G, H: 纹沼螺; I,J : 中华沼螺

Fig. 6 Distribution patterns of densities and biomasses of five dominant $\mathrm{c}$ groups in the Reserve A, B: A. longicornis; C, D: B. aeruginosa: E, F: B. sowerbyi; G, H: P. striatulus; I, J: P. sinensis.

响 ${ }^{[2,15,16]}$. 与之相比, 洞庭湖保护区则常年水位波动较大, 水深成为影响底栖动物分布的首要环境因子, 众 多研究已显示, 水深是影响底栖动物的一个重要因子 ${ }^{[17,18]}$. 一般认为, 水深的变化可导致多种依赖水深的 环境因子发生相应的变化, 从这层意义上说, 水深是这些因子的 “描述器” ( descriptor) ${ }^{[19,20]}$. 而硬度可解释 保护区底栖动物主要以软体动物为优势类群. 研究表明, 在钙离子匮乏的水体常常影响软体动物壳的形成 和骨骼的生长, 在繁殖期间, 软体动物常常趋向高钙的水域 ${ }^{[21-24]}$. 比较而言, 软体动物比寡毛类和水生昆 虫对酸性水体更加敏感 ${ }^{[22]}$ 

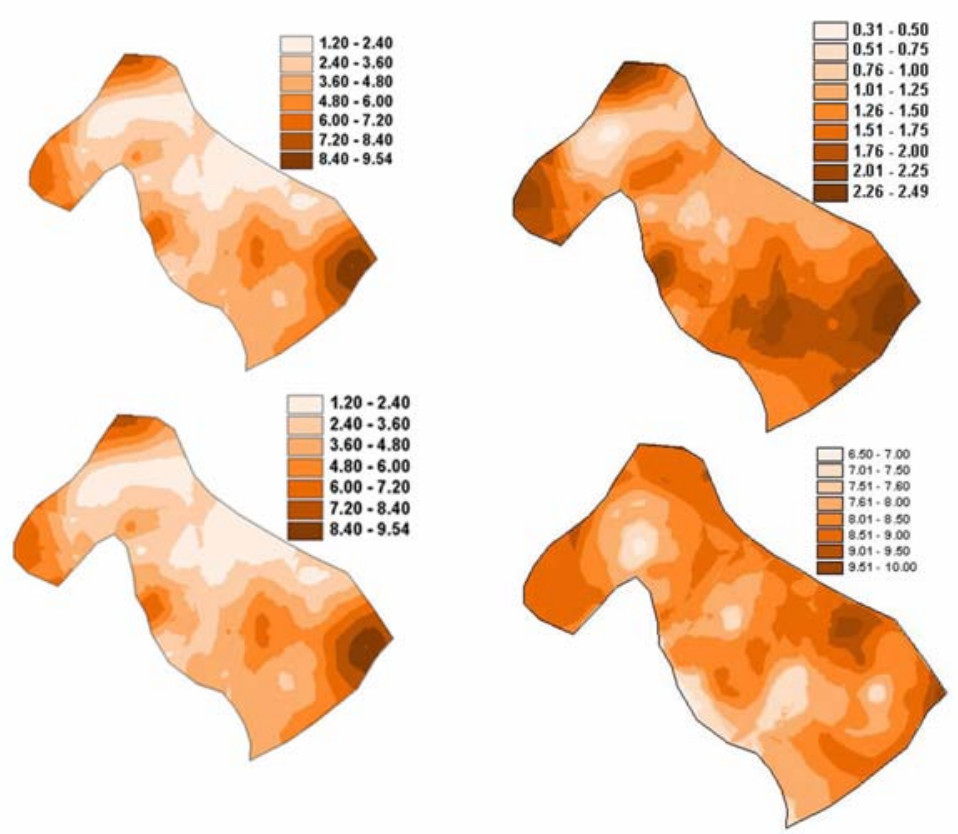

图 7 保护区底栖动物生物多样性指数及生物指数分布格局

Fig. 7 Distribution pattern of Shannon index and Biological Index in the Reserve

大型底栖动物由于具有生命周期长、对环境变化敏感等特点, 是人为干扰和污染监测的重要指示生物 类群,起水下哨兵的作用. 本研究应用 Hilsenhoff' s 生物指数评价保护区的有机污染状况,结果表明,保护 区的有机污染较严重, 模拟的 $B I$ 指数分布图, 较直观的反映保护区的污染分布, 其中西南部的深水区域、北 部及东南部的局部区域水质相对较好, 而东北部的沿岸带大部分水体及南部近六门闸的部分区域水体水质 相对较差.

本研究在系统调查东洞庭湖保护区底栖动物群落结构的基础上,应用 GIS 构建底栖动物的空间分布格 局,应用 GIS 的空间分析功能,初步解析底栖动物与水体理化因子的关系,结果表明, 水深是影响保护区底 栖动物分布格局的关键因子. 构建保护区有机污染的 $B I$ 指数分布图, 较为直观的反映保护区的有机污染 状况. 这些研究, 为更广泛应用 GIS 描述淡水底栖动物的时空分布、精确描述格局与尺度的关系及应用底 栖动物评价水体健康等将有更大的应用前景.

\section{5 参考文献}

[1] 中华人民共和国林业部. 东洞庭湖国家级自然保护区管理计划. 北京: 中国林业出版社,1997.

[2] 王苏民,窦鸿身. 中国湖泊志. 北京:科学出版社, 1998.

[3] Frazier S. A directory of wetlands of international importance. Wetlands International and Ramsar Convention Bureau. Compact Disc. Wetlands International Global Series 13, 2002.

[4] 戴友芝, 唐受印, 张建波. 洞庭湖底栖动物种类分布及水质生物学评价. 生态学报, 2000, 20(2):277 -282 .

[5] Wetzel R G. Limnology ( $2^{\text {nd }}$ edition). Philadelphia: Saunders College Publishing, 1983:1 - 462.

[6] Armitage P, Cranston P S, Pinder C V. The Chironomidae, the biology and ecology of non-biting midges. London: Chapman \& Hall, 1995 : 1 -572.

[7] Morse J C, Yang L, Tian L. Aquatic insects of China useful for monitoring water quality. Nanjing: Hohai University Press, 1994. 
[8] Hilsenhoff W L. An improved biotic index of organic stream pollution. Great Lakes Entomologist, 1987, 30 : $31-39$.

[9] Hilsenhoff W L. Rapid field assessment of organic pollution with a family level biotic index. Journal of the North American Benthological Society, 1988, 7(1): 65 - 68 .

[10] Xie Z C, Tang T, Ma K et al. Influence of environmental variables on macroinvertebrates in a macrophytedominated Chinese lake, with emphasis on the relationships between macrophyte heterogeneity and macroinvertebrate patterns. Journal of Freshwater Ecology, 2005, 20(3) : 503 - 512.

[11] Cyr H, Downing J A. The abundance of phytophilous invertebrates on different species of submerged macrophytes. Freshwat Biol, 1988, 20:365 - 374.

[12] Crowder L B, Cooper W E. Habitat structural complexity and the interaction between bluegills and their prey. Ecology, 1982, 63: $1802-1813$.

[13] Wiggins G B. Larvae of the North America caddisfly Genera ( Trichoptera). Toronto: University of Toronto Press, 1978.

[14] Nolte U, Hoffmann T. Life cycle of Pseudodiamesa branickii (Chironomidae) in a small upland stream. Netherlands Journal of Aquatic Ecology, 1993, 26: 309 - 314.

[15] Nelson W G. Experimental studies of selective predation on amphipods: consequences for amphipod distribution and abundance. Journal of Experimental Marine Biology, 1979, 38: 225 - 245.

[16] Coen L D, Hech K L, Abele L G. Experiments on competition and predation among shrimps of seagrass meadows. Ecology, 1981, 62: $1484-1494$.

[17] Giovanni M, Goretti E, Tamanti V. Macrobenthos in Montedoglio Reservoir, Central Italy. Hydrobiologia 1996, 321: $17-28$.

[18] Brinkhurst R O. The Benthos of Lakes. London: the MaCmillan Press, 1974,1 - 190.

[19] Real M, Riradevall M, Prat N. Chironomus species (Diptera: Chironomidae) in the profundal benthos of Spanish reservoirs and lakes: factors affecting distribution patterns. Freshwater Biology, 2000, 43: 1 - 18.

[20] Oug E. Relation species pattern and environmental variables by canonical ordination: an analysis of soft-bottom macrofauna in the region of Troms, Northern Norway. Marine Environmental Research, 1998, 45(1): $29-45$

[21] Tipping E, Bass J, Haworth E et al. Biological responses to the reversal of acidification in surface waters of the English Lake District. Environmental Pollution, 2002, 116: 137 - 146.

[22] Mcheuhammer M, Mcnicol D, Mallory M et al. Relationships between lake chemistry and alcium trace metal concentrations of aquatic invertebrates eaten by breeding insectivorous waterfowl. Environmental Pollution, $1997,96(2): 235-247$.

[23] Graveland J, Van der Wal R, van Balen J et al. Poor reproduction in forest passerines from decline of snail abundance on acidified siols. Nature, 1994, 368: $446-448$.

[24] Schubauer-Berigan M, Monson P, West C et al. Influence of $\mathrm{pH}$ on the toxicity of Ammonia to Chironomus tentans and LumbriculUs Variegatus. Environmental Toxicology and Chemistry, 1995, 14(4) : 713 - 717. 